\title{
Adaptación de Tres Variables Contextuales en Estudiantes Universitarios Argentinos
}

\author{
Mauricio Federico Zalazar-Jaime ${ }^{1}$, Marcos Cupani \\ Universidad Nacional de Córdoba, Córdoba, Argentina \\ Ana Estefania Azpilicueta \\ Universidad de Palermo, Buenos Aires, Argentina
}

\section{RESUMEN}

Una de las dificultades que han presentado los estudios destinados a identificar las causas de deserción universitaria, es la falta de consideración de variables sociales/contextuales que, según se ha demostrado en la literatura, se relacionan con el proceso de progresión de la carrera. Para la evaluación de dichas variables, el primer paso consiste en la adaptación de pruebas que permitan su medición. Por lo cual, el objetivo del presente trabajo consistió en la adaptación de las escalas de Apertura a la Diversidad, Percepción de Prejuicios y Discriminación y Experiencias Académicas. En este estudio participaron 1295 estudiantes que se encontraban cursando diferentes carreras en la Universidad Nacional de Córdoba (Argentina). Los resultados indicaron valores satisfactorios de consistencia interna $(\rho>.80)$ y se logró replicar la estructura interna de cada instrumento mediante análisis factorial confirmatorio. El estudio de validez indicó que las correlaciones observadas entre las diferentes escalas fueron las esperadas a nivel teórico. Se destacan limitaciones y se sugieren nuevas líneas de estudio.

Palabras clave: Adaptación; Apertura a Diversidad; Percepción de Prejuicios y Discriminación; Experiencias Académicas; Estudiantes Universitarios.

\section{RESUMO - Adaptação de Três Variáveis Contextuais em Estudantes Universitários Argentinos}

Uma das dificuldades que têm apresentado os estudos destinados a identificar as causas de desistência universitária é a falta de consideração de variáveis sociais/contextuais que, segundo se demonstrou na literatura, relacionam-se com o processo de progressão da carreira. Para a avaliação dessas variáveis, o primeiro passo consiste na adaptação de provas que permitam sua medida. Pelo qual, o objetivo do presente trabalho consistiu na adaptação das escalas de Abertura à Diversidade, Percepção de Preconceitos e Discriminação e Experiências Acadêmicas. Neste estudo, participaram 1295 estudantes que se encontravam cursando diferentes carreiras na Universidade Nacional de Córdoba (Argentina). Os resultados indicaram valores satisfatórios de consistência interna $(\rho>.80)$ e conseguiu-se replicar a estrutura interna da cada instrumento mediante análise fatorial confirmatório. O estudo de validade indicou que as correlações observadas entre as diferentes escalas foram as esperadas a nível teórico. Destacam-se, ainda, as limitações e sugerem-se novas linhas de estudo.

Palavras-chave: adaptação; abertura à diversidade; percepção de preconceito e discriminação; experiências acadêmicas; estudantes universitários.

\section{ABSTRACT - Adaptation of Three Contextual Variables in Argentine University Students}

One of the difficulties presented by studies aimed at identifying the causes of university failure is the lack of consideration of social/ contextual variables that, as literature has shown, are related to the process of career progression. To evaluate these variables, the first step is the adaptation of tests that allow their measurement. The purpose of this study was to adapt the Openness to Diversity, Perception of Prejudice and Discrimination, and Academic Experiences scales. This study involved 1,295 students attending different colleges at the National University of Cordoba (Argentina). The results indicated satisfactory values of internal consistency ( $\rho>.80$ ) and it was possible to replicate the internal structure of each instrument through a confirmatory factor analysis. The validity study indicated that the correlations observed between the different scales were as expected at the theoretical level. The limitations are highlighted and new lines of study are suggested.

Keywords: Adaptation; Openness to Diversity; Perception of Prejudice and Discrimination; Academic Experiences; University students.

En las últimas décadas, el porcentaje de estudiantes de nivel superior que abandonan sus estudios ha aumentado paulatinamente, convirtiéndose en un tópico de preocupación para diferentes instituciones educativas y organismos estatales de América Latina (Instituto Internacional para la Educación Superior en América y 
el Caribe, 2006). Particularmente en Argentina, según las últimas cifras oficiales del año 2010, se estima que el fenómeno de deserción universitaria asciende al 59.6\% (Secretaria de Políticas Universitarias, 2010). Según los registros de la Universidad Nacional de Córdoba (UNC), una de las principales instituciones de educación superior de dicho país, en el periodo 2000-2010 el $37.3 \%$ de los alumnos terminaron sus estudios de grado, mientras que el restante $62.7 \%$ los abandonaron, destacando además que de cada diez estudiantes que ingresan, solo egresan cuatro (Fernández, 2013).

En la literatura se han destacado numerosas variables asociadas con el abandono de los estudios universitarios (Araque, Roldan \& Salguero, 2009; Pintos, 2012), entre las cuales se encuentran la dificultad de integración social de los estudiantes (Forbes \& Wickens, 2005), falta de capacidades necesarias para hacer frente a las exigencias académicas (Lightsey, 2006; Saunders, Davis, Williams, \& Williams, 2004), deficiencias pedagógicas por parte de los docentes al momento de transferir los contenidos y establecer relaciones académicas con los alumnos (Lessard, Fortín, Joly, Royer, \& Blaya, 2004), ausencias de soportes sociales tales como el apoyo afectivo, cohesión familiar (Lessard et al., 2008) y ayuda económica de los padres (Fortín, Marcotte, Potvin, Royer, \& Joly, 2006).

Como mencionan algunos trabajos (Lent, Brown, \& Hackett, 2000; Okagaki, 2006; Woolfolk, 2010), el proceso de progresión de la carrera no depende exclusivamente de las capacidades cognoscitivas del estudiante, también intervienen factores contextuales y sociales, como las experiencias que poseen los estudiantes en función de las instituciones educativas a las cuales asisten (Mohr, Eiche, \& Sedlacek, 1998). Particularmente, la teoria Social Cognitiva de la Carrera (Lent, Brown, \& Hackett, 1994) destaca que las influencias contextuales ayudan a determinar como las creencias de autoeficacia, expectativas de resultado, intereses y metas se desarrollaran. Por ejemplo, se postula que las personas son menos propensas a traducir sus intereses de carrera en metas y sus metas en acciones, cuando perciben que sus esfuerzos se ven entorpecidos por factores ambientales adversos (por ejemplo, sistemas de apoyo inadecuados). Por el contrario, la percepción de los factores ambientales beneficiosos (por ejemplo, amplio apoyo y pocos obstáculos/barreras) puede aumentar las creencias de los estudiantes respecto a sus propias capacidades, incentivar las expectativas y facilitar el proceso de traducción de los intereses propios a las metas y éstas en acciones (v.g. Ferry, Fouad \& Smith, 2000; Lent et al., 2001).

Como destacan Nora \& Cabrera (1996), los constructos de Apertura a la Diversidad y Experiencias Académicas han evidenciado contribuciones sobre el rendimiento académico mediante las creencias de autoeficacia, expectativas de resultado y el soporte académico. La Apertura a la Diversidad es entendida como las interacciones y actitudes positivas que posee el estudiante respecto a sus pares de distinta cultura (Pascarella, Edison, Nora, Hagedorn \& Terenzini, 1996), mientras que las Experiencias Académicas enfatizan las relaciones con el personal de la institución educativa y el rol que desempeñan éstos en el desarrollo académico de los estudiantes (Nora \& Cabrera, 1996). Por otro lado, las actitudes (prejuicio) y comportamientos (discriminación) injustificados hacia un individuo (o grupo de personas que comparten características similares en cuanto a la raza o clase social, por ejemplo), se relacionan inversamente con los constructos previamente mencionados (Wright, Aron, McLaughlin-Volpe, \& Ropp, 1997).

Diversos estudios han destacado que los estudiantes universitarios durante sus años de formación generan una mayor apertura, tolerancia e interacciones interpersonales positivas, como por ejemplo sentirse aceptado y valorado (Pascarella et al., 1996; HombradosMendieta \& Castro-Travé, 2013; Felner et al., 1995). Estos aspectos influyen en los alumnos y generan un mayor nivel de confianza, interés y motivación (Roberts et al., 1999; Schmitt, Oswald, Friede, Imus, \& Merritt, 2008), lo cual permite incrementar las probabilidades de retención académica (Nora \& Cabrera, 1996; Dorsey \& Jackson 1995; Fouad \& Bingham; 1995; Woolfolk, 2010), particularmente la de aquellos estudiantes que presentan algún tipo de dificultad emocional o conductual (Hombrados-Mendieta \& Castro-Travé, 2013; Felner et al., 1995).

En relación a las medidas destinadas a evaluar los constructos mencionados, Pascarella et al. (1996) desarrollaron la escala de Apertura a la Diversidad y Nora \& Cabrera (1996) las escalas de Experiencias Académicas y Percepción de Prejuicios y Discriminación. La primera de ellas resulta de una modificación de la escala Disfrute de la Diversidad y Desafío Intelectual (Pascarella et al., 1994), la cual evalúa el placer de ser intelectualmente cuestionado por diferentes ideas, valores y perspectivas, así como las interacciones que el estudiante mantiene con sus pares. Por su parte, la escala de Experiencias Académicas indaga las experiencias e interacciones (formales e informales) de los estudiantes respecto al personal docente y no docente de la institución educativa en cuanto al rol que éstos desempeñan en el desarrollo académico de los estudiantes. Por último, la escala de Percepción de Prejuicios y Discriminación evalúa la presencia de dichas conductas en diferentes ámbitos académicos. En líneas generales, los tres instrumentos poseen resultados satisfactorios de consistencia interna (coeficiente Alfa de Cronbach), y evidencia de estructura interna (análisis factorial exploratorio y confirmatorio).

Como se destacó previamente, la deserción en las universidades públicas de Argentina es una problemática que requiere de estudios que permitan identificar sus causas, y posteriormente, poner en marcha estrategias 
para disminuir su incidencia. Debido a que en nuestro medio no se cuenta con escalas específicas que permitan medir los constructos asociados a la persistencia académica, el objetivo de este trabajo consiste en adaptar las escalas de Apertura a la Diversidad, Percepción de Prejuicios y Discriminación y Experiencias Académicas, lo cual permitirá brindar una contribución al instrumental evaluativo empleado por educadores y psicólogos, y además identificar los factores sociales y contextuales más influyentes, según la literatura, en la obstaculización del progreso académico.

\section{Método}

\section{Participantes y Procedimiento}

En este estudio participaron 1295 estudiantes de los cuales $940(72.6 \%)$ eran de sexo femenino y $316(24.4 \%)$ de sexo masculino, con edades comprendidas entre los 17 y 65 años $(M=22.09 ; D S=5.95)$. Especificamente, 917 estudiantes se encontraban cursando las diferentes carreras de grado dictadas en la Universidad Nacional de Córdoba (UNC, Argentina), mientras que el grupo restante $(N=378)$ se encontraban realizando el curso de ingreso a la facultad de Psicología. La administración de los cuestionarios se desarrolló de manera colectiva y en horario regular de clases. Previo a la administración se explicó el objetivo del estudio en lenguaje claro y comprensible. Se destacó la libre voluntad de participar y la confidencialidad de la información obtenida. Además, se brindó a cada estudiante una nota de consentimiento informado donde los participantes dejaron constancia explícita sobre su interés en participar.

\section{Instrumentos}

Apertura a la Diversidad (Pascarella et al., 1996). Esta escala está compuesta por ocho ítems, los cuales indagan sobre la apertura de los estudiantes respecto a la diversidad cultural y racial. Además, incluye enunciados respecto a la posibilidad de ser desafiado intelectualmente por diferentes ideas, valores y perspectivas. Los participantes deben responder ante ítems como "disfruto tener discusiones con personas que tienen ideas y valores diferentes a los míos", mediante una escala tipo Lickert con cinco opciones de respuesta, entre 1 "totalmente en desacuerdo" y 5 "totalmente de acuerdo". Este instrumento posee estudios de consistencia interna (alfa de Cronbach $\alpha=.83$ ) y evidencia de estructura interna (Pascarella et al., 1994).

Percepción de Prejuicios y Discriminación (Nora \& Cabrera, 1996). Esta escala está compuesta por siete ítems, los cuales indagan la percepción de prejuicio y discriminación en tres ámbitos: campus universitario (e.g., "he observado palabras, comportamientos o gestos discriminatorios dirigidos a estudiantes de grupos minoritarios en esta facultad"); personal de la facultad (e.g., "siento que hay un clima general de prejuicio entre los profesores en esta institución”), y desarrollo de la clase (e.g. "he sido señalado en clases y tratado de un modo diferente al resto de los estudiantes"). En su versión original (Cabrera \& Nora, 1994) este instrumento cuenta con estudios de consistencia interna (alfa de Cronbach $\alpha>.80$ ) en población minoritaria y no-minoritaria.

Experiencias Académicas (Nora \& Cabrera, 1996). Esta escala está compuesta por 11 ítems los cuales indagan sobre las experiencias (cinco ítems, e.g., "la mayoría de los miembros de la facultad con los que tengo contacto están verdaderamente interesados en enseñar") e interacciones (seis ítems, e.g., "estoy contento con la oportunidad de conocer e interactuar informalmente con los miembros de la facultad") entre los estudiantes y el personal docente y no docente de la institución académica y el rol que desempeñan éstos en el desarrollo académico de los estudiantes. Los participantes deben responder utilizando una escala tipo Lickert con cinco opciones de respuesta entre 1 "totalmente en desacuerdo" y 5 "totalmente de acuerdo". En un trabajo desarrollado por Nora \& Cabrera (1996), los autores reportaron estudios de consistencia interna (alfa de Cronbach, $\alpha=.80$ para experiencia y $\alpha=.84$ para interacción) y evidencia de estructura interna (análisis factorial exploratorio y confirmatorio).

\section{Análisis de Datos}

En primer lugar, se realizó una traducción (método directo) del inglés al español, de los ítems de las diferentes escalas. Esta actividad fue realizada por nueve expertos en la lengua inglesa, tres por cada instrumento. A partir de la versión traducida se procuró mantener cierta equivalencia conceptual, semántica y funcional más que una mera traducción literal de la versión original (Mimura \& Griffiths, 2008). Posteriormente, los datos para los análisis propuestos se prepararon a través del software SPSS para Windows versión 19.0. A continuación, se evaluó el patrón de los valores perdidos considerando los ítems de cada instrumento con el fin de estimar si estos respondían a una distribución aleatoria (Tabachnick \& Fidell, 2011). Se calculó la media, desviación estándar, asimetría y curtosis para cada escala. Para evaluar los índices de asimetría y curtosis se utilizó el criterio que estipula que valores comprendidos entre \pm 1.00 se consideran excelentes, mientras que los valores inferiores a \pm 2.00 , adecuados (George \& Mallery, 2011). Se identificaron los casos atípicos univariados mediante el cálculo de puntuaciones z para cada variable (puntuaciones $z> \pm 3.29$ fueron considerados atípicos).

Se condujo un análisis factorial confirmatorio (AFC) con el fin de replicar las estructuras originales de cada instrumento. Se utilizó el software estadístico Mplus versión 6.12, ya que permite aplicar el método de estimación denominado mínimos cuadrados ponderados (WLS), considerado el más oportuno cuando se trata de datos categóricos (escalas Likert, por ejemplo; Flora \& Curran, 2004). Para evaluar el ajuste de los modelos, se utilizaron los estadísticos Chi-Cuadrado, el índice de 
Ajuste Comparativo (CFI), el índice de Tucker-Lewis (TLI), el Error Cuadrático Medio de Aproximación (RMSEA), y la ponderada Media Cuadrática Residual (WRMR). Los valores entre .90 y .95 o superiores para el CFI y TLI son considerados como ajustes aceptables a excelentes, mientras que se esperan valores entre .05 y .08 para el RMSEA, y valores menores a 1.00 para WRMR (Yu \& Muthén, 2002).

Como destacan Cortina (1993) y Raykov (1998) el empleo del estadístico alfa de Cronbach como indicador de consistencia interna ha sido criticado en la literatura debido a su dependencia con el número de ítems de la escala y las correlaciones entre ellas. Por lo tanto, se estimó la confiabilidad compuesta $(\rho)$. Este método utiliza las cargas factoriales, varianza residual y $R^{2}$ de cada ítem según una estructura subyacente, presentando de esta manera una menor variación del error. Valores iguales o superiores a $\rho=.70$ son considerados como aceptables (Nunnally, 1978). En base a los objetivos y análisis propuestos en este trabajo, la presentación de los datos se desarrolló en cuatro fases, una por cada instrumento, mientras que en la cuarta fase se desarrolla el estudio de validez entre los diferentes constructos.

\section{Resultados}

\section{Traducción de los Ítems}

En el proceso de adecuación de los ítems, algunas expresiones como "academic advisors or counselors" de la escala de experiencia académica, fueron sustituidas por "directivos de esta facultad (docentes y no docentes)" ya que, en primer lugar, en el contexto universitario argentino no existe la figura del consejero (counselor), es decir, una persona encargada del asesoramiento y orientación profesional de los estudiantes. En segundo lugar, la figura del asesor (academic advisors) puede ser desempeñada por los docentes y en menor medida por la figura no docente (personal a cargo de la función administrativa). Por ejemplo, los docentes generalmente brindan sugerencias a los alumnos sobre modalidades para abordar el estudio de un determinado tema, Cabrera que personal no docente auxilia en cuanto a aspectos vinculados a la resolución de problemas burocráticos (inscripción a cursadas o superposición de exámenes).

\section{Apertura a la Diversidad}

Preparación de los datos y análisis de ítems. Los casos perdidos oscilaron entre $2.2 \%$ (ítem 1, por ejemplo) a $2.6 \%$ (ítem 7 ). Al no superar el $5 \%$ establecido (Schafer, 1999) se procedió a imputar los datos por el modo. Respecto a la asimetría, se observó que seis ítems presentaron valores entre \pm 1.00 y dos ítems presentaron valores entre \pm 2.00 . Por su parte, los estudios sobre la curtosis revelaron que cinco ítems presentaron valores entre \pm 1.00 y tres ítems presentaron valores entre \pm 2.00 (ver Tabla 1). En cuanto a los casos atípicos, se identificaron 58 casos univariados y 54 casos multivariados. Si bien la presencia de casos atípicos tiende a distorsionar los resultados, en este caso se decidió retenerlos con el fin de evitar la limitación en la generalización de los resultados, debido a que se dejaría de representar a un segmento de la población en estudio (Hair, Anderson, Tatham, \& Black, 1999).

Análisis factorial confirmatorio. El ajuste a los datos no fue adecuado (CFI .917, TLI .883 RMSEA .128, 90\% CI .118 .139, WRMR 2.125). Los pesos de regresión estandarizados $(p \leq .05)$ oscilaron entre .58 a .74 . Debido a que el ajuste no fue óptimo, según los criterios propuestos, se decidió inspeccionar los índices de modificación (IM). Cuando los residuos se encuentran altamente correlacionados se considera que existe redundancia debido a la superposición de contenidos (Brown, 2015). El análisis de estos IM indicó una ganancia del ajuste al correlacionar los residuos entre los pares de ítems seis-ocho y cuatro-siete. Por lo tanto, considerando que se trata de un solapamiento por la semejanza respecto al contenido, y con el fin de conservar los ítems originales del instrumento, se decidió correlacionar ambos ítems. De este modo, el ajuste mejoró levemente presentando un ajuste adecuado $(\mathrm{CFI}=0,959, \mathrm{TLI}=0,936$, RMSEA $=0,095,90 \% \mathrm{CI}=0,084$ 0,106, WRMR=1,457).

Consistencia interna. Se reportó un valor satisfactorio $(\rho=.84)$ para la escala de Apertura a la Diversidad.

Tabla 1

Media, Desviación Estándar, Asimetría, Curtosis y Pesos Estandarizados de la Escala Apertura a la Diversidad

\begin{tabular}{lccccc}
\hline & $M$ & $D E$ & Asimetría & Curtosis & $\beta$ \\
\hline Ítem 1 & 4,01 & 0,96 & $-1,08$ & 1,20 &, 61 \\
Ítem 2 & 3,98 & 0,82 & $-0,73$ & 1,00 &, 59 \\
Ítem 3 & 4,17 & 0,79 & $-0,91$ & 1,26 &, 76 \\
Ítem 4 & 4,20 & 0,80 & $-0,89$ & 0,80 &, 69 \\
Ítem 5 & 3,72 & 0,98 & $-0,49$ & $-0,09$ &, 63 \\
Ítem 6 & 4,27 & 0,82 & $-1,10$ & 1,13 &, 63 \\
Ítem 7 & 3,83 & 0,99 & $-0,61$ & 0,03 &, 58 \\
Ítem 8 & 4,16 & 0,86 & $-0,86$ & 0,54 &, 54 \\
\hline
\end{tabular}

Nota. $M=$ media; $D E=$ desviación estándar; $\beta=$ peso beta estandarizado 


\section{Percepción de Prejuicios y Discriminación}

Preparación de los datos y análisis de ítems. Los casos perdidos oscilaron entre $2.7 \%$ (ítem 4) a 3.4\% (ítem 2 , por ejemplo). Al no superar el $5 \%$ establecido (Schafer, 1999) se procedió a imputar los datos por el modo. Respecto a la asimetría, se observó que dos ítems presentaron valores entre \pm 1.00 , cuatro ítems presentaron valores entre \pm 2.00 , y solo un ítem presentó un valor $> \pm 2.00$. En cuanto a la curtosis, tres ítems presentaron valores entre \pm 1.00 , un ítem presentó valores entre \pm 2.00 , y tres ítems presentaron valores $> \pm 2.00$ (ver Tabla 2). En cuanto a los casos atípicos, se identificaron 98 casos univariados y 81 casos multivariados, los cuales se decidió mantener.
Análisis factorial confirmatorio. El AFC indicó un ajuste adecuado a los datos (CFI $=0,952$, TLI $=0,928$ RMSEA $=0,187,90 \%$ CI $=0,175$ 0,199, WRMR $=3,024)$. Los pesos de regresión estandarizados $(p \leq .05)$ oscilaron entre .66 a .90. Los índices de modificación indicaron un solapamiento entre los pares de ítems cinco-seis y seis-siete. Siguiendo los mismos criterios, se correlacionaron los ítems mencionados. De este modo, el ajuste del modelo mejoró levemente (CFI $=0,989$, TLI $=0,980$, RMSEA $=0,098,90 \% \mathrm{CI}=0,084$ 0,111, WRMR $=1,177$ ).

Confiabilidad compuesta. El estudio de consistencia interna indicó un valor satisfactorio $(\rho=.91)$ para la escala Percepción de Prejuicios y Discriminación.

Tabla 2

Media, Desviación Estándar, Asimetría, Curtosis y Pesos Estandarizados de la Escala Percepción de Prejuicios y Discriminación

\begin{tabular}{lccccc}
\hline & $M$ & $D E$ & Asimetría & Curtosis & $\beta$ \\
\hline Ítem 1 & 2,06 & 1,08 & 0,72 & $-0,17$ &, 81 \\
Ítem 2 & 2,22 & 1,05 & 0,62 & $-0,14$ &, 79 \\
Ítem 3 & 1,60 & 0,92 & 1,71 & 2,73 &, 87 \\
Ítem 4 & 1,56 & 0,92 & 1,73 & 2,58 &, 83 \\
Ítem 5 & 1,75 & 0,99 & 1,22 & 0,87 &, 71 \\
Ítem 6 & 1,69 & 0,95 & 1,34 & 1,23 &, 71 \\
Ítem 7 & 1,28 & 0,68 & 2,71 & 7,33 &, 69 \\
\hline
\end{tabular}

Nota. $M=$ media; $D E=$ desviación estándar; $\beta=$ peso beta estandarizado

\section{Experiencias Académicas}

Preparación de los datos y análisis de ítems. Los casos perdidos oscilaron entre $0.6 \%$ (ítem 5 , por ejemplo) a 2.4\% (ítem 2). Al no superar el 5\% establecido (Schafer, 1999) se procedió a imputar los datos por el modo. Respecto a la asimetría y curtosis, se observó que los 20 ítems presentaron valores entre \pm 1.00 (ver Tabla 3). En cuanto a los casos atípicos, se identificaron tres casos univariados y 12 casos multivariados, los cuales se decidió mantener.

Análisis factorial confirmatorio. $\mathrm{El} \mathrm{AFC}$ no indicó un ajuste adecuado a los datos (CFI $=0,892$, $\mathrm{TLI}=0,862$, RMSEA $=0,136,90 \% \quad \mathrm{CI}=0,125 \quad 0,148$,
WRMR $=1,856)$. Los pesos de regresión estandarizados $(p \leq .05)$ para el factor experiencia oscilaron entre .44 a .87 , y para el factor interacción, entre .45 a .77. Los índices de modificación indicaron un solapamiento entre los pares de ítems 10-11 y 9-11. En función de estos resultados, y debido a la semejanza entre los ítems mencionados en lo referente al contenido, se optó por correlacionarlos. De este modo, el ajuste del modelo mejoró levemente logrando un ajuste adecuado (CFI $=0,934$, TLI $=0,911$, RMSEA $=0,109,90 \% \mathrm{CI}=0,907$ 0,121, WRMR $=1,455)$.

Consistencia interna. Los valores obtenidos fueron satisfactorios, experiencia $\rho=.82$, interacción $\rho=.83$.

Tabla 3

Media, Desviación Estándar, Asimetría, Curtosis, Pesos Estandarizados e Índices de Ajuste de la Escala Experiencias Académicas

\begin{tabular}{|c|c|c|c|c|c|c|}
\hline & M & $D E$ & Asimetría & Curtosis & $\beta$ factor 1 & $\beta$ factor 2 \\
\hline Ítem 1 & 3,85 & 0,93 & $-0,86$ & 0,72 & ,60 & - \\
\hline Ítem 2 & 3,74 & 0,93 & $-0,67$ & 0,45 & ,44 & - \\
\hline Ítem 3 & 3,79 & 0,95 & $-0,63$ & 0,19 & ,71 & - \\
\hline Ítem 4 & 3,68 & 0,95 & $-0,60$ & 0,33 & ,79 & - \\
\hline Ítem 5 & 3,91 & 0,83 & $-0,55$ & 0,18 & ,87 & - \\
\hline Ítem 6 & 3,38 & 1,11 & $-0,45$ & $-0,44$ & - & ,77 \\
\hline Ítem 7 & 3,41 & 0,93 & $-0,31$ & 0,15 & - & ,75 \\
\hline Ítem 8 & 3,24 & 1,02 & $-0,28$ & $-0,26$ & - & ,75 \\
\hline Ítem 9 & 3,43 & 0,80 & $-0,23$ & 0,53 & - & ,65 \\
\hline Ítem 10 & 3,16 & 0,98 & $-0,14$ & 0,03 & - & ,45 \\
\hline Ítem 11 & 2,99 & 1,24 & $-0,03$ & $-0,96$ & - & ,51 \\
\hline
\end{tabular}

Nota. $M=$ media; $D E=$ desviación estándar; $\beta$ =peso beta estandarizado; Factor 1=Experiencia; Factor 2=Interacción 


\section{Estudio de Validez}

Se obtuvo la matriz de correlaciones entre las escalas de Percepción de Prejuicios y Discriminación, Apertura a la Diversidad y Experiencias Académicas (ver Tabla 4). El factor Experiencia, el cual refiere a actitudes positivas y de interés entre el estudiante y su entorno académico próximo, presentó una correlación negativa $(r=-.30)$ con la Percepción de Prejuicios y Discriminación y positiva con Apertura a la Diversidad $(r=.16)$. Además, ésta última escala también presentó una correlación positiva y moderada con la sub-escala
Interacción $(r=.30)$. En líneas generales los resultados son consistentes por lo reportado en la literatura. Es decir, una percepción positiva con respecto a la experiencia académica de los estudiantes indica que ellos mismos no perciben indicios de prejuicios y discriminación. Además, como indican Nora y Cabrera (1996), el interés y preocupación de los estudiantes y profesores por el progreso académico (Experiencia) y los vínculos positivos generados entre ellos (Interacciones) posibilita que los estudiantes estén abiertos a otras perspectivas diferentes a la propia (Apertura a la Diversidad).

Tabla 4

Matriz de Correlaciones entre las Escalas de Percepción de Prejuicios y Discriminación, Apertura a la Diversidad y Experiencias Académicas

\begin{tabular}{|c|c|c|c|c|}
\hline & 1 & 2 & 3 & 4 \\
\hline 1. Experiencia & 1,00 & , $44^{* *}$ &,$- 30^{* *}$ & $16^{* *}$ \\
\hline 2. Interacción & & 1,00 &,- 06 & $30^{* *}$ \\
\hline 3. Percepción de Prejuicios y Discriminación & & & 1,00 & 01 \\
\hline 4. Apertura a la Diversidad & & & & 1,00 \\
\hline
\end{tabular}

\section{Discusión}

La deserción universitaria es un fenómeno complejo que aqueja al sistema educativo universitario público argentino. A pesar de la existencia de estudios locales que identifican un conjunto de variables entre las posibles causas del abandono universitario (Landi \& Giuliodori, 2001), generalmente estos estudios se centran en variables cognitivas personales dejando de lado otros factores ambientales de relevancia los cuales indicen en el proceso de progresión de la carrera (Lent, Brown \& Hackket, 2000). Por lo tanto, el objetivo de este trabajo consistió en adaptar las escalas de Apertura a la Diversidad, Percepción de Prejuicios y Discriminación y Experiencias Académicas.

En términos generales, las escalas de Apertura a la Diversidad y Percepción de Prejuicios y Discriminación presentaron adecuadas propiedades psicométricas. Los estudios de consistencia interna evidenciaron resultados levemente superiores a los reportados por los estudios originales, lo cual puede deberse al estimador utilizando en ambos trabajos. Es decir, Pascarella et al. (1996) y Nora \& Cabrera (1996) emplearon el coeficiente Alfa de Cronbach el cual tiende a sobreestimar o subestimar las estimaciones, mientras que el estadístico de confiabilidad compuesta presenta una menor variación del error (ver Raykov, 1998 para una revisión).

Los resultados del análisis factorial confirmatorio lograron replicar la estructura original de un factor de las escalas de Percepción de Prejuicios y Discriminación y Apertura a la Diversidad, y de dos factores para la escala de Experiencias Académicas. Si bien estos resultados sugieren que las escalas propuestas son viables en una muestra de estudiantes universitarios argentinos, es importante realizar nuevos estudios de validez de contenido (entrevistas cognitivas). Respecto al estudio de validez, las correlaciones observadas entre las diferentes escalas fueron las esperadas a nivel teórico, sin embargo los coeficientes de correlación observados fueron de una magnitud débil a moderada. Si bien era esperable una asociación negativa entre la Percepción de Prejuicios y Discriminación y la Apertura a la Diversidad, en este trabajo no se observó una correlación entre ambas variables.

Respecto a las limitaciones del trabajo, se deben tomar en cuenta algunas consideraciones en relación a la muestra. Por un lado, debe considerarse el hecho de que estuvo compuesta por un gran porcentaje de mujeres. Esta desproporción podría haber distorsionado las experiencias e historial de interacciones que poseen los estudiantes de grado respecto de su entorno académico. Si bien no existen antecedentes que destaquen diferencias entre ambos sexos, se ha observado que en ciertos constructos como soporte social, los hombres y las mujeres tiendan a prestar una atención diferencial al soporte percibido por distintas fuentes como padres y amigos (por ejemplo, Zalazar-Jaime \& Cupani, 2016).

Otra limitación reside en que gran parte de los participantes se encontraban cursando carreras vinculadas al área de ciencias sociales y humanidades (por ejemplo, psicología, comunicación social y artes), y en menor proporción alumnos del área de ciencias naturales (física e ingeniería, por ejemplo). Si bien esta desproporción no podría afectar los resultados, es importante considerar todas las áreas del conocimiento debido a que las características de las variables en estudio se encuentran 
íntimamente ligadas a las percepciones de los jóvenes respecto de su unidad académica. Por otra parte, sería importante contemplar otras variables teóricamente asociadas a estos constructos, con el fin de obtener evidencia de validez externa.

Además, es importante realizar una serie de entrevistas cognitivas, técnica la cual posibilita la obtención de información sobre qué es lo que está pensando el sujeto al momento de responder un cuestionario, permitiendo una mejor comprensión, contextualización, orientación temporal y especificidad de los ítems (Ajzen, 1988; Almond et al., 2009). Esto es fundamental debido a que los test psicológicos se desarrollan en un contexto particular, por tanto el uso de los mismos en un medio diferente al cual fue diseñado puede acarrear una serie de problemas, los cuales no se agotan en el proceso de adaptación, en cuanto a la familiaridad de los estímulos y aspectos ligados con la muestra (por ejemplo, nivel educativo).
En conclusión, a pesar del carácter preliminar de los resultados del presente estudio, se obtuvieron nuevos instrumentos adaptados al medio local que permiten un correcto registro y evaluación de las experiencias académicas, las cuales son sumamente relevantes en la predicción de la persistencia académica en estudiantes universitarios. Además, estas medidas permitirán evaluar nuevos modelos explicativos de persistencia académica, según el marco establecido por la teoría social cognitiva de la carrera (Lent, Brown, \& Hackett, 1994). En relación a las aplicaciones prácticas, la utilización de estos instrumentos por parte de los gabinetes de seguimiento o pedagógicos de cada unidad académica, posibilitarán el diseño de estrategias de intervención, con el fin de identificar a aquellos jóvenes que perciban a su entorno académico de manera poco favorable, es decir, con mayores niveles de prejuicios, discriminación y experiencias académicas negativas, que puedan entorpecer el proceso de progresión de carrera.

\section{Referencias}

Ajzen, I. (1988). Attitudes, personality, and behavior. Chicago: Dorsey Press.

Almond, P., Cameto, R., Johnstone, C. J., Laitusis, C., Lazarus, S., Nagle, K., Parker, C. E., Roach, A. T., \& Sato, E. (2009). White paper: Cognitive interview methods in reading test design and development for alternate assessments based on modified academic achievement standards (AAMAS). Dover, NH: Measured Progress and Menlo Park, CA: SRI International.

Araque, F., Roldán, C., \& Salguero, A. (2009). Factors influencing university drop out rates. Computers \& Education, 53(3), 563-574. doi: 10.1016/j.compedu.2009.03.013

Brown, T. A. (2015). Confirmatory factor analysis for applied research. New York: Guilford Publications.

Cabrera, A. F., \& Nora, A. (1994). College students' perceptions of prejudice and dis- crimination and their feelings of alienation. Review of Education, Pedagogy, and Cultural Studies, 16(1994), 387-409. doi: 10.1080/1071441940160310

Cortina, J. M. (1993). What is coefficient alpha? An examination of theory and applications. Journal of applied psychology, 78(1), 98. doi: 10.1037/0021-9010.78.1.98

Dorsey, M. S., \& Jackson, A. P. (1995). Afro-American students' perceptions of factors affecting academic performance at a predominantly white school. The Western Journal of Black Studies, 19(3), 189-195.

Felner, R. D., Brand, S., Dubois, D. L., Adan, A. M., Mulhall, P. F., \& Evans, E. G. (1995). Socioeconomic disadvantage, proximal environmental experiences and socio-emotional and academic adjustment in early adolescence: investigation of a mediated effects model. Child Development, 66, 774-792. doi: 10.2307/1131950

Fernández, M. (2013, 10 de febrero). De cada 10 que ingresan, egresan cuatro. La Voz del Interior. Retrieved from http://www.lavoz.com.ar/ cordoba/cada-10-que-ingresan-egresan-cuatro.

Flora, D. B., \& Curran, P. J. (2004). An empirical evaluation of alternative methods of estimation for confirmatory factor analysis with ordinal data. Psychological methods, 9(4), 466-491. doi: 10.1037/1082-989X.9.4.466

Forbes, A. \& Wickens, E. (2005). A good social live helps students to stay the course. Times High Education Supplement, 1676, 58-63.

Fortín, L., Marcotte, D., Potvin, P., Royer, E., \& Joly, J. (2006). Typology of student at risk of dropping out of school: Description by personal, family and school factors. European Journal of Psychology of Education, 21(4), 363-383. doi: 10.1007/BF03173508

Fouad, N. A., \& Bingham, R. P. (1995). Career counseling with racial and ethnic minorities. In Walsh, B, Savickas, M. L. \& Hartung, P. J. (Eds). Handbook of vocational psychology: Theory, research, and practice (pp. 331-365). New York: Practice Routledge.

George. D., \& Mallery. P. (2011). IBM SPSS Statistics 21 step by step: A simple guide and reference (13th ed.). Boston: Pearson Education.

Hair, J., Anderson, R., Tatham, R., \& Black, W. (1999). Análisis Multivariante (4ª edición). España: Prentice Hall.

Hombrados-Mendieta, I., \& Castro-Travé, M. (2013). Apoyo social, clima social y percepción de conflictos en un contexto educativo intercultural. Anales de Psicología, 29(1), 108-122. doi: 10.6018/analesps.29.1.123311

Instituto Internacional para la Educación Superior en América y el Caribe (2006). Informe sobre la Educación Superior en América Latina y el Caribe 2000-2005. La Metamorfosis de la Educación Superior. Caracas: UNESCO.

Landi, J., \& Giuliodori, R. (2001). Graduación y deserción en las Universidades Nacionales en estudiantes y profesionales de Argentina. Comunicación presentada en el Encuentro Nacional: La problemática del Ingreso. Córdoba: Universidad Nacional de Córdoba.

Lent, R. W., Brown, S. D., \& Hackett, G. (1994). Toward a unifying social cognitive theory of career and academic interest, choice, and performance. Journal of vocational behavior, 45(1), 79-122. doi: 10.1006/jvbe.1994.1027

Lent, R. W., Brown, S. D., \& Hackett, G. (2000). Contextual supports and barriers to career choice: A social cognitive analysis. Journal of Counseling Psychology, 47(1), 36-49. doi: 10.1037/0022-0167.47.1.36 
Lessard, A., Butler-Kisber, L., Fortin Marcotte, D., Potvin, P., \& Royer, É. (2008). Shadows disengagement: high school dropouts speak out. Social Psychology of Education, 11(1), 25-42. doi: 10.1007/s11218-007-9033-z

Lessard, A., Fortin, L., Joly, J., Royer, É. \& Blaya, C. (2004). Students at-risk for dropping out of school: are there gender differences among personal, family and school factors? Journal of At-Risk Issues, 10(2), 91-127. Retrieved from http://dropoutprevention.org/wp-content/ uploads/2015/05/2004JARI1002.pdf

Lightsey, O. R. (2006). Resilience, meaning and well-being. Counseling Psychologist, 34(1), 96-107. doi: 10.1177/0011000005282369

Mimura, C., \& Griffiths, P. (2008). A Japanese version of the Perceived Stress Scale: Cross-cultural translation and equivalence assessment. BMC psychiatry, 8(1), 85. doi: 10.1186/1471-244X-8-85.

Mohr, J. J., Eiche, K., \&Sedlacek, W. E. (1998). So close, yet so far: predictors of attrition in college seniors. Journal of College Student Development, 39, 343-353. Retrieved from http://williamsedlacek.info/publications/articles/soclose1.pdf

Nora, A., \& Cabrera, A. (1996). The role of perceptions of prejudice and discrimination on the adjustment of minority students to college. Journal of Higher Education, 67(2), 119-148. doi: 10.2307/2943977

Nunnally, J. C. (1978). Psychometric theory (2nd ed.). New York: McGraw-Hill.

Okagaki, L. (2006). Ethnicity, learning. En P. Alexander y P. Winne (eds.), Handbook of educational psychology (2a ed., pp. 615-634). Mahwah, NL: Erlbaum.

Pascarella, E. T., Edison, M., Nora, A., Hagedorn, L. S., \& Terenzini, P. T. (1996). Influences on students' openness to diversity and challenge in the first year of college. The Journal of Higher Education, 67(2), 174-195. doi: 10.2307/2943979

Pascarella, E., Bohr, L., Nora, A., Ranganathan, S., Desler, M., \& Bulakowski, C. (1994). Impacts of 2-year and 4-year colleges on learning orientations: A preliminary study. Community College Journal of Research and Practice, 18(6), 577-589. doi: 10.1080/1066892940180607

Pintos, M. E. (2012, 26 de marzo). En el primer año, el 58\% de los estudiantes dejan o cambian de carrera. Clarín.com, Sociedad. Retrieved from http://www.clarin.com/sociedad/primer-estudiantes-dejancambian-carrera_0_670732977.html

Raykov, T. (1998). Coefficient alpha and composite reliability with interrelated nonhomogeneous items. Applied psychological measurement, 22(4), 375-385. doi: 10.1177/014662169802200407

Roberts, R., Phinney, J., Masse, L., Chen, Y., Roberts, C., \& Romero, A. (1999). The structure of ethnic identity of young adolescents from diverse ethnocultural groups. Journal of Early Adolescence, 19(3), 301-322. doi: 10.1177/0272431699019003001

Saunders, J., Davis, L., Williams, T., \& Williams, J.H. (2004). Gender differences in self-perceptions and academic outcomes: a study of African American high school students. Journal of Youth and Adolescence, 33(1), 81-91. doi: 10.1023/A:1027390531768

Schafer, J.L. (1999). Multiple imputation: a primer. Statistical Methods in Medical Research, 8(1), 3-15. doi: 10.1177/096228029900800102

Schmitt, N., Oswald, F. L., Friede, A., Imus, A., \& Merritt, S. (2008). Perceived fit with an academic environment: Attitudinal and behavioral outcomes. Journal of Vocational Behavior, 72(3), 317-335. doi: 10.1016/j.jvb.2007.10.007

Secretaria de Políticas Universitarias-Ministerio de Educación (2010).Anuario 2010. Estadísticas Universitarias. Retrieved from http://repositorio. educacion.gov.ar/dspace/bitstream/handle/123456789/109591/Anuario_de_Estadisticas_Universitarias_2010.pdf?sequence=1

Tabachnick. B. G., \& Fidell. L. S. (2011). Using Multivariate Statistics (6th ed.). Needham Heights: Pearson.

Woolfolk, A. (2010). Psicología Educativa. Mexico: Prentice-Hall.

Wright, S. C., Aron, A., McLaughlin-Volpe, T., \& Ropp, S. A. (1997). The extended contact effect: Knowledge of cross-group friendships and prejudice. Journal of Personality and Social psychology, 73(1), 73. doi: 10.1037/0022-3514.73.1.73

Yu, C. Y., \& Muthen, B. (2002, April). Evaluation of model fit indices for latent variable models with categorical and continuous outcomes. In Annual meeting of the American Educational Research Association, New Orleans, LA.

Zalazar-Jaime, M. F. \& Cupani, M. (2016). Adaptación de dos Medidas de Apoyo Social en una Muestra de Estudiantes Universitarios Argentinos. Actualidades en Psicología, 30(120), 57-70. doi: 10.15517/ap.v30i120.21809

\section{Sobre os autores}

Mauricio Federico Zalazar-Jaime és Licenciado en Psicología por la Universidad Nacional de Córdoba. IIPsi, Instituto de Investigaciones Psicológicas. [CONICET y UNC]. Su tema de investigación consiste en determinar cómo ciertos factores contextuales, sociales y cognitivos contribuyen a explicar la persistencia académica en estudiantes universitarios.

Marcos Cupani és Doctor en Psicología por la Universidad Nacional de Córdoba. Es investigador adjunto en el CONICET y profesor Profesor Titular Cátedra de Técnicas Psicométricas I, Facultad de Psicología, Universidad Nacional de Córdoba. Dirige tesis de doctorado y coordina proyectos de investigación en el IIPsi, Instituto de Investigaciones Psicológicas. [CONICET y UNC]. Sus investigaciones se centran en la educación superior, el rendimiento académico y los instrumentos de evaluación.

Ana Estefania Azpilicueta és Licenciada en Psicología por la Universidad Siglo 21. Actualmente se desempeña como becaria doctoral de CONICET en el IIPsi, Instituto de Investigaciones Psicológicas. [CONICET y UNC]. 